\title{
Confinement Transitions (H-mode) in JET inner wall limiter plasmas
}

D. Borba ${ }^{1}$, B. Alper ${ }^{3}$, G. D. Conway ${ }^{2}$, I. Nunes ${ }^{1}$, S. Hacquin ${ }^{1}$, D.C. McDonald ${ }^{3}$, G. Maddison ${ }^{3}$, P. Lomas ${ }^{3}$, S.D. Pinches ${ }^{2}$ and the EFDA-JET contributors.

${ }^{1}$ Centro de Fusão Nuclear, Associação EURATOM/IST, Instituto Superior Técnico, Av Rovisco Pais, 1049-001 Lisboa, Portugal ${ }^{2}$ MPI für plasmaphysik, EURATOM-Association, D-85748 Garching, Germany

${ }^{3}$ EURATOM/UKAEA Fusion Association, Culham Science Centre, OX14 3DB, UK.

\section{Introduction}

It is foreseen that the next step tokamak fusion experiment (ITER) will operate in improved confinement regimes such as the H-mode [F. Wagner et al]. H-mode is characterised by a spontaneous confinement transition that leads to an increase in plasma density and temperature, when the plasma heating power exceeds a given threshold [M.Shimada, et.al.,]. H-mode confinement is obtained mostly in divertor plasmas [J A Snipes et al], i.e. in configurations with the magnetic separatrix inside the vacuum vessel, either two ("double null configuration") or one ("single null configuration") poloidal field nulls [A. Tanga et al]. Transitions to $\mathrm{H}$-mode have been also observed in limiter plasma configurations. These observations have been reported in several tokamaks, such as TFTR [Bush, C. E et al,1994,1995], TEXT-U [D R Roberts, et al] and JIPP T-IIU [K. Toi et al] Tokamaks. H-mode confinement transitions have been also recently observed in JET inner wall limiter plasmas and the confinement properties of these plasmas are analysed in this paper.

\section{Limiter H-mode in JET}

Confinement transitions with the characteristics of H-mode are observed in JET inner wall limited plasmas in experiments performed at magnetic field of $0.8 \mathrm{~T}$ and at a current of 0.9 MA, using 5MW of auxiliary heating power. The NBI heating is switch on for a period of 3 seconds (Figure 1), which is sufficient to achieve quasi steady state, given that both the overall energy confinement time and the beam slowing down time in these discharges are less than 200ms. During this period several abrupt transitions in confinement are observed, which are characterised by a significant decrease in the $\mathrm{H}$-alpha emission (Figure 2). These confinement transitions are short lived, with duration of up to $20 \mathrm{~ms}$, but the overall stored energy increases by up to $5 \%$ and edge density by up to $20 \%$ (Figure 2). In the Ohmic phase of the discharge, the L-mode confinement time is $300 \mathrm{~ms}$. The confinement time in the L-mode phase with 5MW of NBI auxiliary power is around 100-150ms. The value of the stored energy measured by the diamagnetic loop under these conditions is $0.8 \mathrm{MJ}$ and it is modulated by the Sawtooth activity (Figure 1). The increase in plasma stored energy during the $\mathrm{H}$-mode transition is around $30-40 \mathrm{~kJ}(5 \%)$ in approximately $10-15 \mathrm{~ms}$. This transient increase in the plasma stored energy can be explained by a $50 \%$ reduction of the power loss, which corresponds to a transient increase of up to $200 \%$ in confinement time, i.e. to around $300 \mathrm{~ms}$. 

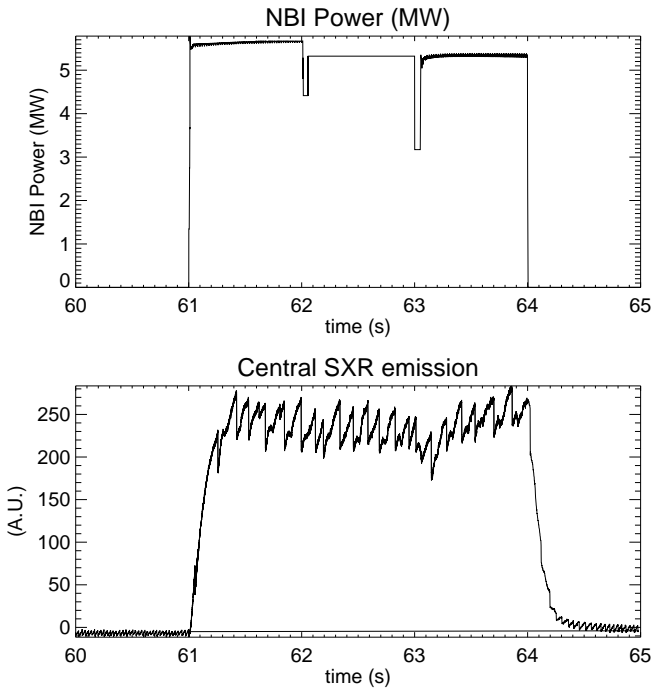

Figure 1. Time evolution of NBI power for the limiter discharge \#60908 and the central soft $x$-rays emission showing the Sawtooth.
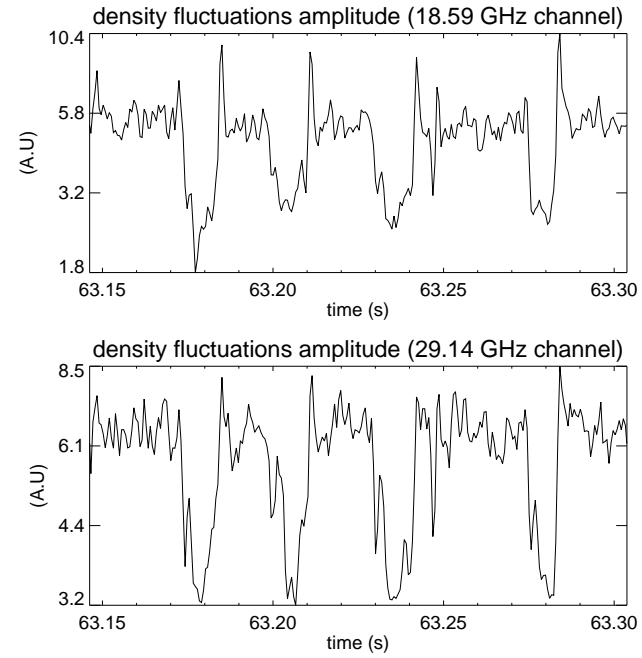

Figure 3. The overall spectrum of density fluctuation measured by the microwave reflectometer channels $18.59 \mathrm{GHz}$ and 29.14 $\mathrm{GHz}$ (Shot \#60908), probing the plasma edge.
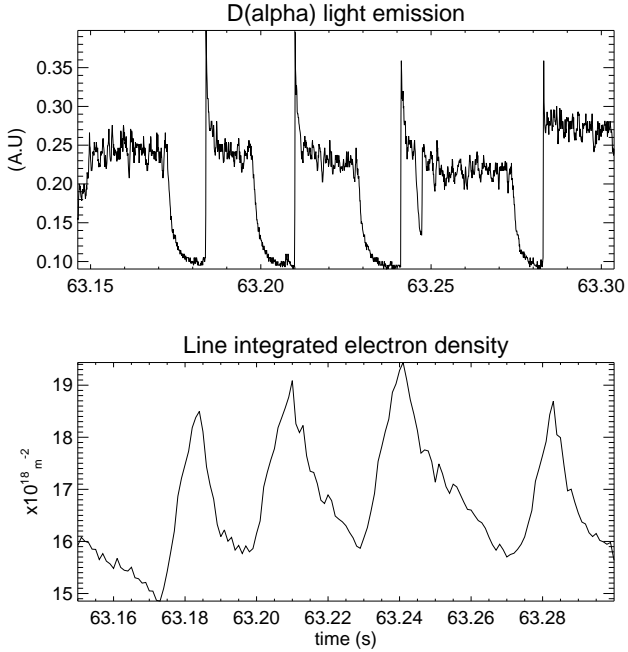

Figure 2. D-alpha light emission and edge density (microwave interferometer) evolution during the H-mode transitions.

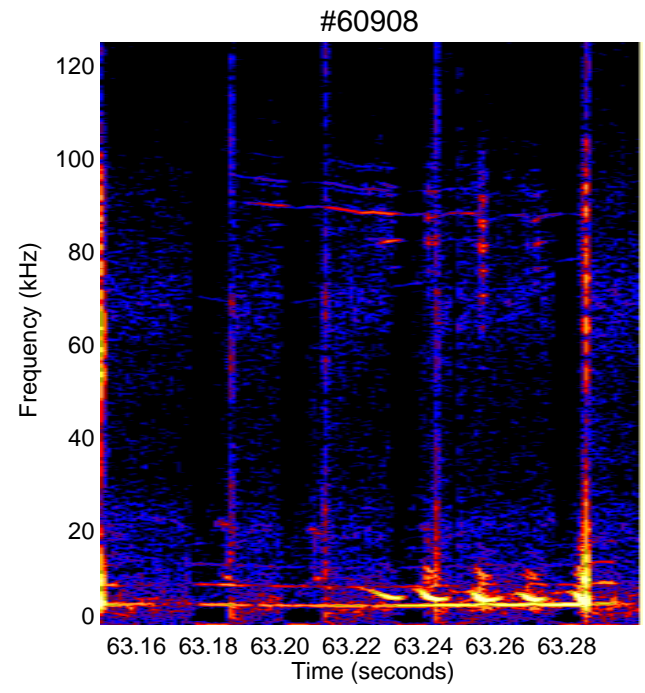

Figure 4. Spectrogram of magnetic fluctuations (Mirnov coils), shows no correlation between h-mode transitions and (sawtooth, fishbones or TAE) in Shot \#60908.

\section{Fluctuations Spectra}

These transitions in confinement correlate also with a significant decrease in the fluctuations measured by the microwave reflectometer probing the plasma edge (Figure 3) [G.D.Conway, 1997] and magnetic sensors (Figure 4), accompanied by a reduction of the D-alpha emission (Figure 2). The density measurements using Thompson scattering shows a very flat density profile, with a maximum value of $(2-3) \times 10^{19} \mathrm{~m}^{-3}$. The plasma edge fluctuations measurements are performed using two fixed frequency $(18,29 \mathrm{GHz}) \mathrm{O}$-mode reflectometer channels, probing the plasma edge with densities $(0.5-1.0) \times 10^{19} \mathrm{~m}^{-3}$. The comparison of the fluctuations in the transitions to limiter H-mode in JET with similar 
discharges (same current, magnetic field and auxiliary heating power) in divertor (x-point) configuration shows a similar behaviour, as shown in figure 5 (limiter) and figure 6 (xpoint). In particular, the reduction of the fluctuation levels at high frequencies $(f>10 \mathrm{kHz})$ and an increase in the fluctuation levels at lower frequencies ( $\mathrm{f}<10 \mathrm{kHz}$ ). In both cases the fluctuation measurements are performed just before $(10 \mathrm{~ms})$ and just after the L-H transition (10ms). Thus, the density, temperature and rotation profiles have not evolved significantly during $20 \mathrm{~ms}$ and can be assumed to be the same. Therefore, the changes in the fluctuation levels can be attributed to changes in the turbulence levels.

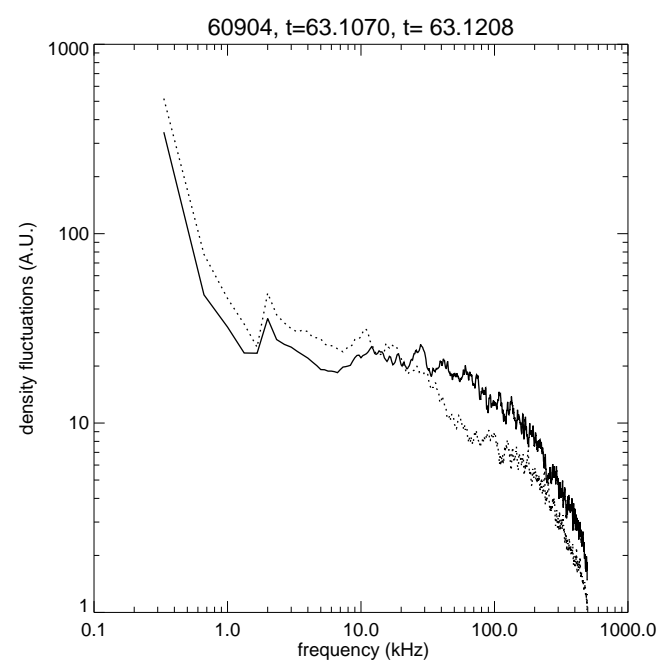

Figure 5. Spectrum of density fluctuations from $18 \mathrm{GHz}$ reflectometer just before(10ms) the H-mode transition (solid line) and just (10ms) after the transition (dashed line) in limiter configuration.
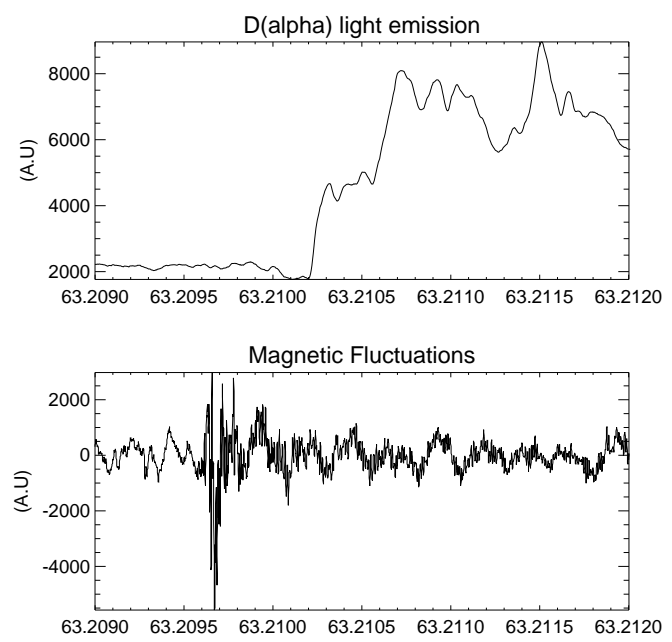

Figure 7. D-alpha emission and magnetic fluctuations showing a burst of magnetic activity before the termination of the period of good confinement (limiter configuration \#60908)

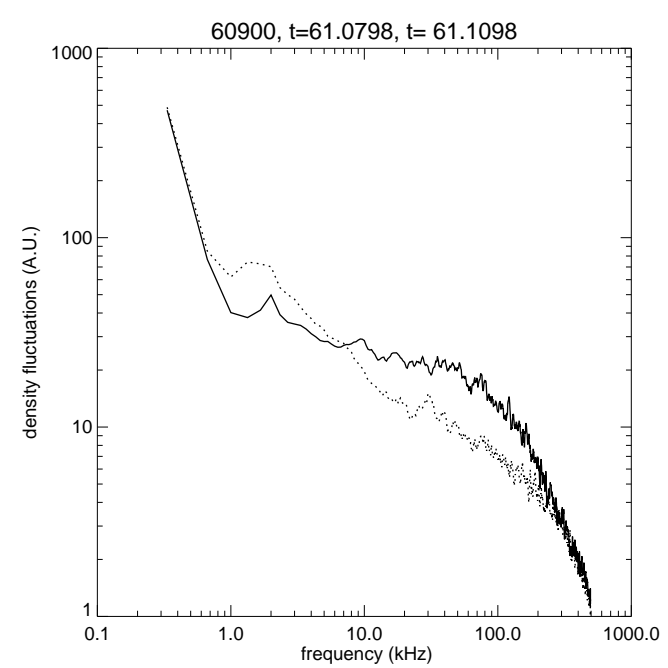

Figure 6. Spectrum of density fluctuations from $18 \mathrm{GHz}$ reflectometer before the H-mode transition (solid line) and after the transition (dashed line) in $x$-point configuration.
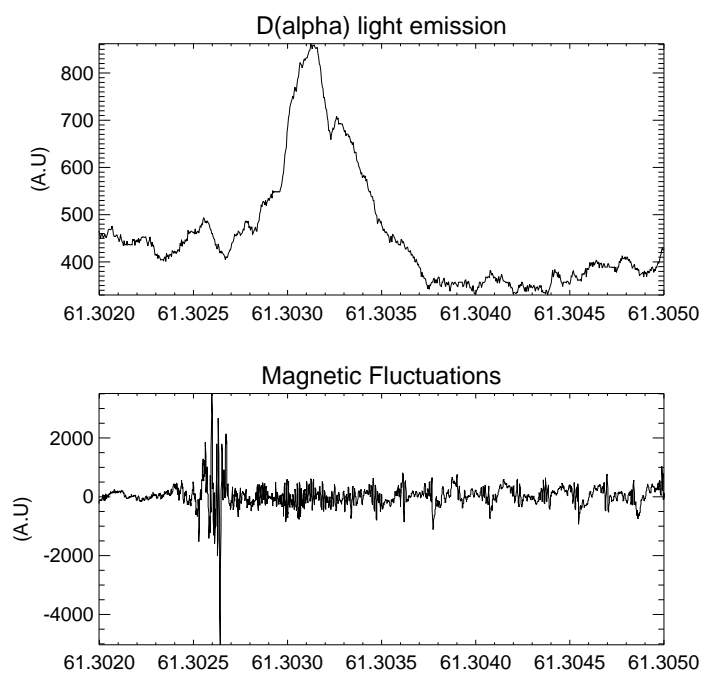

Figure 8. D-alpha emission and magnetic fluctuations showing a burst of magnetic activity and an increase in the D-alpha during a Edge Localised Mode (ELM) event (x-point configuration \#60900) 


\section{Termination of the good confinement period}

The termination of the period of good confinement correlates with the observation of a burst of magnetic fluctuations (Figure 7), similar to those associated with Edge Localised Modes (ELMs) (Figure 8) for the same plasma current, magnetic field and auxiliary heating power. A 500 microseconds delay between the maximum in the magnetic activity and the sudden increase in the D-alpha light is observed in both cases.

\section{Conclusions}

JET inner wall limiter configurations at low field and current show confinement transitions with the same characteristics of H-modes i.e. increase in density and stored energy, accompanied with a decrease in D-alpha and in the density fluctuations (with similar spectral characteristics for similar plasma parameters plasma current, magnetic field and auxiliary heating power). However, limiter H-modes in JET are short lived $(<20 \mathrm{~ms})$ and they are terminated by a burst of magnetic fluctuations with very similar characteristics to those of ELMs. The onset of the good confinement period is caused by a reduction in micro-turbulent transport very close to the plasma edge. On the other hand the termination of the period of good confinement has a clear magnetic signature, most likely caused by an edge instability driven by the steep pressure or current profiles.

\section{Acknowledgements}

This work, carried out under the European Fusion Development Agreement, supported by the European Communities and "Instituto Superior Técnico", has been carried out within the Contract of Association between EURATOM and IST. Financial support was also received from "Fundação para a Ciência e Tecnologia" in the frame of the Contract of Associated Laboratory. The views and opinions expressed herein do not necessarily reflect those of the European Commission, IST and FCT.

\section{References}

Bush, C. E et al, Plasma Physics and Controlled Fusion, Volume 36, Issue 7A, pp. A153A158 (1994)

C. E. Bush, Physics of Plasmas Vol 2(6) pp. 2366-2374. June 1995

G.D. Conway, $3^{\text {rd }}$ International Reflectometry Workshop for fusion plasma diagnostics Madrid $5^{\text {th }} / 7^{\text {th }}$ of May 1997, Informe Técnica CIEMAT 838, p39.

D R Roberts, et al Plasma Phys. Control. Fusion 38 (August 1996) 1117-1125, [5] N Bretz et al, Plasma Phys. Control. Fusion 36 (July 1994) A141-A14.

M.Shimada, et.al., IAEA-CN-77/ITERP/05.

J A Snipes et al, Plasma Phys. Control. Fusion 42 (May 2000) A299-A308

A. Tanga et al, Proceedings of the Eleventh International Conference on Plasma Physics and Controlled Fusion Research, Kyoto,1986 (Ref 3), p 65.

K. Toi et al Phys. Rev. Lett. 64, 1895 (1990)

F. Wagner et al., Phys. Rev. Lett. 49, 1408 (1982) 